\title{
DATOS HISTÓRICOS Y BIBLIOMÉTRICOS DEL CORPUS DE LUIS VÉLEZ DE GUEVARA PARA LA FECHA DE ESCRITURA DE EL ALBA Y EL SOL
}

\author{
JAVIER J. GONZÁLEZ MarTínez \\ Escuela Superior de Arte Dramático de Castilla y León
}

No hemos encontrado ningún dato concreto que nos informe objetivamente sobre el momento de escritura de El alba y el sol. ${ }^{1}$ Spencer y Schevill mencionan como fecha ad quem el año 1617 pero no explican las razones que les llevan a fijar dicho momento ni citan la fuente de la que pudieron obtener el dato. ${ }^{2}$

\footnotetext{
${ }^{1}$ Recibido: 1/II/2011 Aceptado: 31/III/2011

Esta pieza puede encontrarse también con los nombres de La restauración de España, o El Alba de los Asturias y el Sol de los vizcaínos. Para las referencias a manuscritos, sueltas y adaptaciones modernas remito a la reciente edición de El alba y el sol, con partituras de Antonio Guerrero y Enrique Moreno, a cargo de William R. Manson y C. George Peale, Delaware, Juan de la Cuesta, 2010, pp. 56-62.

2 Cfr. F. E. Spencer y R. Schevill, The dramatics works of Luis Vélez de Guevara. Their plots, sources, and bibliography, Berkeley, University of California Press, 1937, p. 145.
} 
Por eso trataremos de acotar la fecha a través de la aplicación del método que Morley y Bruerton ${ }^{3}$ crearon para la datación de las comedias de Lope de Vega según la métrica utilizada. ${ }^{4}$ Adaptamos esta herramienta a las obras de Luis Vélez de Guevara basándonos en las piezas que ya están fechadas y tienen edición moderna. Salen un total de treinta obras que abarcan todo su periodo productivo sin apenas considerables saltos en el tiempo: cotejamos piezas desde 1602 hasta 1634 donde el intervalo más grande entre una y otra es de cinco años (1628-1633).

En primer lugar recogemos la distribución de los distintos metros a lo largo de El alba y el sol en el siguiente esquema: ${ }^{5}$

${ }_{3}$ Cfr. S. G. Morley y C. Bruerton, Cronología de las comedias de Lope de Vega, versión española de M. R. Cartes, Madrid, Gredos, 1968.

4 Vid. M. Zugasti, «Estudio introductorio», en Las palabras a los reyes y gloria de los Pizarros, Delaware, Juan de la Cuesta, 2004, p. 84-85. Estoy de acuerdo con Zugasti cuando afirma que el método métrico es poco infructuoso cuando se utiliza en comparaciones Vélez-Lope o Vélez-Tirso. Por eso se hace necesario un estudio crono-métrico completo del ecijano que formule datos concretos dentro de su propia obra sin tener que compararla con otros.

5 Cfr. G. Peale, «Estudio bibliográfico», en El alba y el sol, de Luis Vélez de Guevara, Delaware, Juan de la Cuesta, 2010, pp. 68-69. 
Datos históricos y bibliométricos del corpus de Luis Vélez de Guevara para la fecha de escritura de El alba y el sol

\begin{tabular}{|l|c|c|}
\hline \multicolumn{1}{|c|}{ MÉTRICA } & VERSOS & ACTO \\
\hline Silva de consonantes & $1-76$ & \\
\hline Seguidilla & $77-82$ \\
\hline Romance o-o & $83-138$ \\
\hline Silva de consonantes & $139-156$ \\
\hline Quintilla & $157-161$ \\
\hline Romance o-o & $162-178$ \\
\hline Seguidilla & $179-184$ \\
\hline Romance o-o & $185-529$ \\
\hline Silva de consonantes & $530-631$ \\
\hline Romance i-o & $632-809$ \\
\hline Romancillo o-a & $810-823$ \\
\hline Romance o-a & $824-835$ \\
\hline Décimas & $836-895$ \\
\hline Romance o-a & $896-913$ \\
\hline Décimas & $914-1093$ \\
\hline Romance o-a & $1094-1433$ \\
\hline Romancillo o-a & $1434-1443$ \\
\hline Romance e-e & $1444-1453$ \\
\hline Romance a-a & $1454-1885$ \\
\hline Romance e-e & $1886-1891$ \\
\hline Octavas & $1892-1939$ \\
\hline Romance i-a & $1940-2119$ \\
\hline Romance a-a & $2120-2139$ \\
\hline Romancillo u-a & $2140-2221$ \\
\hline Redondillas & $2222-2337$ \\
\hline Romance e-a & $2338-2447$ \\
\hline Octavas & $2448-2487$ \\
\hline Romance e-e & $2488-2585$ \\
\hline Redondillas & $2586-2601$ \\
\hline Romance e-o & $2602-2815$ \\
\hline Redondillas & II \\
\hline Romance a-o & III \\
\hline Redondillas & \\
\hline Romance a-e & \\
\hline
\end{tabular}

A continuación, nos detenemos en el uso del romance en distintas obras de Luis Vélez. A medida que avanza el siglo XVII el romance se va imponiendo en el teatro: pasamos de porcentajes mínimos a obras en las que lo raro es encontrar un metro distinto del romance. 


\begin{tabular}{|c|c|c|c|c|c|c|c|}
\hline FECHA & TÍTULO & $\begin{array}{l}\mathrm{ROM} \\
\mathrm{n}^{\mathrm{o}} \mathrm{vv} .\end{array}$ & $\begin{array}{c}\text { PASAJE } \\
\text { más largo }\end{array}$ & $\%$ & $\begin{array}{c}\mathrm{N}^{\mathrm{o}} \\
\text { pasajes }\end{array}$ & $\begin{array}{l}\text { RED } \\
\mathrm{n}^{\mathrm{o}} \mathrm{vv}\end{array}$ & $\%$ \\
\hline $1602-03^{1}$ & El espejo del mundo ${ }^{2}$ & 714 & 232 & 27 & 4 & 1292 & 49 \\
\hline $1604-1610^{3}$ & La devoción de la misa ${ }^{4}$ & 450 & 244 & 14.2 & 5 & 2196 & 69,1 \\
\hline $1606-1610^{5}$ & La obligación a las mujeres ${ }^{6}$ & 616 & 236 & 22,8 & 4 & 1052 & 38,9 \\
\hline $1608-10^{7}$ & Los fijos de la Barbuda & 794 & 184 & 28,7 & 6 & 1184 & 42,8 \\
\hline $1602-1605^{9}$ & La hermosura de Raquel, $I^{10}$ & 388 & 206 & 11,4 & 3 & 1000 & 29,4 \\
\hline $1602-1608^{11}$ & La hermosura de Raquel, III & 380 & 172 & 11,8 & 3 & 1828 & 56,9 \\
\hline Circa $1607^{13}$ & El rey don Sebastián ${ }^{14}$ & 748 & & 24,8 & & 684 & 22,7 \\
\hline $1608-1614^{15}$ & La niña de Gómez Arias ${ }^{16}$ & 594 & 288 & 22,71 & 3 & 1384 & 52,9 \\
\hline $1613^{17}$ & La montañesa de Asturias ${ }^{18}$ & 1078 & 234 & 38 & 6 & 1500 & 52,8 \\
\hline $1613^{19}$ & La serrana de la Vera ${ }^{20}$ & 1012 & 406 & 30,6 & 5 & 1544 & 46,7 \\
\hline $1613^{21}$ & Don Pedro Miago 22 & 1008 & 320 & 31,1 & 6 & 1124 & 42,8 \\
\hline $1615^{23}$ & El amor en vizcaíno ${ }^{24}$ & 1667 & 331 & 61,9 & 7 & 492 & 18,3 \\
\hline $1615^{25}$ & El conde don Pero Vélez $z^{26}$ & 920 & 270 & 29,0 & 9 & 2068 & 65 \\
\hline $1615-17^{27}$ & El conde don Sancho Niño ${ }^{28}$ & 1192 & 260 & 50,9 & 6 & 864 & 36,87 \\
\hline $1617^{29}$ & El caballero del Sol ${ }^{30}$ & 1382 & 342 & 48,0 & 8 & & \\
\hline $1621^{31}$ & Más pesa el rey que la sangre 32 & 1714 & 365 & 68 & 9 & 492 & 19,6 \\
\hline $1621^{33}$ & Virtudes vencen señales ${ }^{34}$ & 1772 & 404 & 38,8 & 6 & 1048 & 35,6 \\
\hline $1621-22^{35}$ & El hijo del águila ${ }^{36}$ & 1286 & 458 & 48,3 & 5 & 1176 & 44,1 \\
\hline $1623^{37}$ & El primer conde de Orgaz ${ }^{38}$ & 328 & 200 & 10,7 & 2 & 1460 & 47,5 \\
\hline $1623^{39}$ & El rey naciendo mujer ${ }^{40}$ & 1916 & 346 & 70,5 & 9 & 520 & 19,1 \\
\hline $1623-24^{41}$ & El asombro de Turquía ${ }^{42}$ & 1058 & 268 & 42,7 & 8 & 1060 & 42,7 \\
\hline $1622-25^{43}$ & El Hércules de Ocaña ${ }^{44}$ & 1338 & 388 & 45 & 6 & 1348 & 45,3 \\
\hline $1625^{45}$ & El rey en su imaginación ${ }^{46}$ & 1540 & 340 & 63,4 & 8 & & \\
\hline $1625^{47}$ & A lo que obliga el ser rey ${ }^{48}$ & 1560 & 412 & 59 & 7 & & \\
\hline $1625 ?-26^{49}$ & Los novios de Hornachuelos ${ }^{50}$ & 1568 & 296 & 50 & 9 & & \\
\hline $1625-27^{51}$ & Si el caballo vos han muerto ${ }^{52}$ & 1538 & 300 & 62 & 8 & 728 & 29,4 \\
\hline $1628^{53}$ & Las palabras a los reyes ${ }^{54}$ & 1259 & 384 & 50,1 & 9 & 864 & 34,3 \\
\hline $1630 ?-39 ?^{55}$ & Reinar después de morir ${ }^{56}$ & 1466 & 266 & 59,2 & 16 & 410 & 16,6 \\
\hline $1633^{57}$ & El águila del agua ${ }^{58}$ & 2511 & 589 & 68,7 & 11 & 1016 & 27,8 \\
\hline $1634^{59}$ & El cerco del Peñón de Vélez ${ }^{60}$ & 730 & 172 & 24,9 & 9 & 1960 & 67,5 \\
\hline
\end{tabular}

\section{(Notas tabla)}

1 Cfr. G. Peale y H. Urzáiz Tortajada, «Vélez de Guevara», en Historia del Teatro Español, Madrid, Gredos, 2003, p. 934.

2 Cfr. G. Peale, «Estudio bibliográfico y métrico», en El espejo del mundo de Luis Vélez de Guevara,

Delaware, Juan de la Cuesta, 2002, pp. 95-96.

3 Cfr. ibidem, p. 253.

4 Cfr. ibidem, p. 249.

5 Cfr. ibidem, p. 253.

${ }^{6}$ Cfr. ibidem, p. 249. 
Datos históricos y bibliométricos del corpus de Luis Vélez de Guevara para la fecha de escritura de El alba y el sol

7 Cfr. ibidem, p. 253.

8 Cfr. ibidem, p. 249.

9 Cfr. ibidem, p. 253.

10 Cfr. ibidem, p. 249.

11 Cfr. ibidem, p. 253.

12 Cfr. ibidem, p. 249.

13 Cfr. G. Peale, «Estudio introductorio», en La mayor desgracia de Carlos Quinto de Luis Vélez de Guevara, Delaware, Juan de la Cuesta, 2002, p. 50.

${ }_{14}$ Cfr. W. Herzog, Introducción y edición de la Comedia famosa del rey don Sebastián, Madrid, Anejos del Boletín de la Real Academia Española, 1972, p. 56.

15 Cfr. R. Rozzell, «Introducción», en La niña de Gómez Arias de Luis Vélez de Guevara, Granada, Universidad de Granada, 1959, p. 60.

16 Cfr. ibidem, pp. 54-55.

17 Cfr. M. G. Hauer, «An addendum to Luis Vélez de Guevara: A critical bibliography», en G. Peale (ed.), Antigüedad y actualidad de Luis Vélez de Guevara: Estudios críticos, Amsterdam, Benjamins, 1983, p. 277.

18 Cfr. G. Peale [2010], «Introducción textual a El alba y el sol», p. 71.

19 Cfr. M. G. Hauer [1983], op. cit., p. 283; G. Peale, «Estudio introductorio», en La mayor desgracia de Carlos Quinto de Luis Vélez de Guevara, Delaware, Juan de la Cuesta, 2002, p. 96; M. G. Hauer, Luis Vélez de Guevara: A critical bibliography, North Carolina, University of North Carolina Press, 1975, p. 163; C. Bruerton, «Eight plays by Vélez de Guevara», Romance Philology, vol. VI, no 4 (mayo 1953), p. 251; E. Cotarelo, «Luis Vélez de Guevara y sus obras dramáticas», BRAE, III (1916), p. 647; R. Menéndez Pidal y M. Goyri de Menéndez Pidal, «Introducción», en La serrana de la Vera, Teatro Antiguo Español, I, Madrid, 1916, pp. 125-127.

20 Cfr. Peale [2002], «Introducción textual a La serrana de la Vera», pp. 49-51; P. Bolaños, «Introducción», La serrana de la Vera de Luis Vélez de Guevara, Madrid, Castalia, 2001, pp. 66-72.

${ }^{21}$ Cfr. Peale y Urzáiz, op. cit., p. 934.

22 Cfr. Bruerton [1953], op. cit., p. 251.

23 Cfr. E. Rodríguez Cuadros, «Introducción», en El amor en vizcaíno, celos en francés y torneos de Navarra de Luis Vélez de Guevara, Delaware, Juan de la Cuesta, 2002, p. 28.

${ }^{24}$ Cfr. Peale [2002], «Introducción métrica y bibliográfica a El amor en vizcaíno, celos en francés y torneos de Navarra», p. 114.

25 Cfr. M. G. Hauer [1975], op. cit., p. 163; E. Cotarelo, «Luis Vélez de Guevara y sus obras dramáticas», BRAE, IV (1917), p. 278; G. Peale, «Estudio introductorio», en El águila del agua de Luis Vélez de Guevara, Delaware, Juan de la Cuesta, 2003, p. 76; Bruerton [1953], op. cit., p. 251.

${ }^{26}$ Cfr. G. Peale, «Estudio introductorio», en El conde don Pero Vélez de Luis Vélez de Guevara, Delaware, Juan de la Cuesta, 2002, pp. 84-86.

${ }^{27}$ Cfr. G. Peale, «Vélez de Guevara contextualizado: una vida singular y su ámbito ético», Bulletin of the Comediantes, vol. 61, n 1 (2009), p. 57.

28 Cfr. R. Bininger y R. L. Landeira, «Introducción», en El Conde don Sancho Niño de Luis Vélez de Guevara, Vigo, Faro de Vigo, 1970, pp. 48-49.

${ }_{29}$ Cfr. G. Peale, «Estudio introductorio», en El águila del agua de Luis Vélez de Guevara, Delaware, Juan de la Cuesta, 2003, p.76; T. Ferrer Valls, La práctica escénica cortesana: de la época del emperador a la de Felipe III, London, Tamesis Book, 1991, p. 179.

30 Cfr. Bininger y Landeira [1970], op. cit., p. 29.

31 Cfr. J. J. González Martínez, «Más pesa el rey que la sangre: el Guzmán de Luis Vélez junto al Quijote y el Cid», en J. Matas Caballero y J. M. Balcells Doménech (eds), D. Pérez Fernández (coord.), Cervantes y su tiempo, Actas del Congreso celebrado en León 2-5 de noviembre de 2005, vol. I, Lectura y Signo, Anejo I, León, Universidad de León, 2008, p. 442.

32 Cfr. F. J. Bianco, «Introducción», en Más pesa el rey que la sangre de Luis Vélez de Guevara, 
Barcelona, Puvill-Editor, 1979, pp. 61-63.

${ }_{33}$ Cfr. M. G. Profeti, «Introduzione, testo critico e note», en Virtudes vencen señales de Luis Vélez de Guevara, Pisa, Universitá di Pisa, 1965, p. 41.

${ }^{34}$ Cfr. ibidem, p. 60.

${ }_{35}$ Cfr. G. Peale, «Estudio introductorio» en El hijo del águila de Luis Vélez de Guevara, Delaware, Juan de la Cuesta, 2003, p. 45.

36 Cfr. ibidem, p. 50.

${ }_{37}$ Cfr. G. Peale, «Estudio introductorio», en La mayor desgracia de Carlos Quinto de Luis Vélez de Guevara, Delaware, Juan de la Cuesta, 2002, p. 50.

38 Cfr. G. Peale [2002], «Estudio introductorio a El primer conde de Orgaz», pp. 40-41.

39 Cfr. G. Peale [2009], op. cit., p. 57.

40 Cfr. G. Peale, «Estudio introductorio», en El rey naciendo mujer de Luis Vélez de Guevara, Delaware, Juan de la Cuesta, 2006, pp. 34-35.

${ }^{41}$ Cfr. J. J. González Martínez, «Estudio introductorio», en El asombro de Turquía de Luis Vélez de Guevara, Delaware, Juan de la Cuesta, 2010, p. 20.

${ }^{42}$ Cfr. G. Peale, «Estudio bibliográfico», en El asombro de Turquía de Luis Vélez de Guevara, Delaware, Juan de la Cuesta, 2010, p. 44.

${ }_{43}$ Cfr. J. J. González Martínez, Tesis doctoral inédita El teatro histórico nacional de Luis Vélez, Universidad de Valladolid, 2007.

${ }^{44}$ Cfr. C. Bruerton, «The date of Schaeffer's Tomo Antiguo», Hispanic Review, XV (1947), p. 362.

${ }^{45}$ Cfr. H. Urzáiz Tortajada, Luis Vélez de Guevara, Teatro Breve, Madrid, Iberoamericana-Vervuert, 2002, p. 24; G. Peale [2009], op. cit., p. 57.

46 Cfr. Bruerton [1947], op. cit., p. 350.

47 Cfr. G. Peale y H. Urzáiz, op. cit., p. 936.

${ }^{48}$ Cfr. Bruerton [1947], op. cit., p. 351.

49 Cfr. Ibidem, p. 351; Bininger y Landeira [1970], op. cit., p. 29.

s0 Cfr. Bruerton [1947], op. cit., p. 350.

${ }_{51}$ Cfr. J. J. González Martínez, «Estudio introductorio», en Si el caballo vos han muerto, y blasón de los Mendozas de Luis Vélez de Guevara, Delaware, Juan de la Cuesta, 2007, p. 19.

${ }_{52}$ Cfr. G. Peale, «Estudio bibliográfico», en Si el caballo vos han muerto, de Luis Vélez de Guevara, Delaware, Juan de la Cuesta, 2007, pp. 38-39.

${ }_{53}$ Cfr. G. Peale, «Estudio introductorio», en La mayor desgracia de Carlos Quinto de Luis Vélez de Guevara, Delaware, Juan de la Cuesta, 2002, p. 50; J. J. González Martínez, «El rey como espectador del teatro indiano: Las palabras a los reyes de Luis Vélez de Guevara», en S. Mattalia, P. Celma, P. Alonso (eds.); A. C. Lafarga, C. Morán Rodríguez (colaboradoras), Actas del VII Congreso Internacional de la AEELH-Universidad de Valladolid, Homenaje a José Luis de la Fuente, «El viaje en la Literatura Latinoamericana: El espíritu colombino», Valladolid 19-22 de septiembre de 2006; Frankfurt am Main, Iberoamericana, Vervuert, 2008, p. 228.

${ }_{54}$ M. Zugasti [2004], op. cit., pp. 85-86.

${ }^{55}$ Cfr. Bruerton [1947], op. cit., p. 352; Bininger y Landeira [1970], op. cit., p. 29.

56 Cfr. G. Peale, «Estudio bibliográfico y métrico», en Reinar después de morir de Luis Vélez de Guevara, Delaware, Juan de la Cuesta, 2008, pp. 51-52.

${ }^{57}$ Cfr. G. Peale [2009], op. cit., p. 57.

58 Cfr. G. Peale, «Estudio introductorio», en El águila del agua de Luis Vélez de Guevara, Delaware, Juan de la Cuesta, 2003, pp. 79-81.

59 Cfr. G. Peale, «Estudio textual y criterios de edición», en El cerco del Peñón de Vélez de Luis Vélez de Guevara, Delaware, Juan de la Cuesta, 2003, p. 47.

${ }^{60}$ Cfr. Ibidem, pp. 51-53. 
El total de versos de El alba y el sol es de 3197. Corresponden a romance 2408, que suponen un $75,4 \%$ del total de versos. Son veinte los pasajes en este metro. El más largo tiene una extensión de 430 versos, lo que supone una cantidad muy elevada si lo comparamos con los datos de otras obras de Luis Vélez. Es la obra del ecijano con un mayor porcentaje de versos en romance dentro del corpus que hemos acotado. También es la que tiene un mayor número de pasajes en ese metro.

La mayoría de las posteriores a 1620 superan el 50\% de versos en romance y el porcentaje crece aún más a partir de $1625 .{ }^{6}$ De todas formas, podemos concluir con los datos que trabajamos de estas treinta piezas que el uso del romance nos marca unas tendencias pero desde luego no es posible utilizarlo para definir una fecha exacta. Observamos que en efecto en la primera década de siglo las cifras están entre el 10 y el 30\%. En la segunda década oscilan mayoritariamente entre el 30 y el 50\%. Y en la tercera década se encuentran entre el 50 y el 70\%. Pero dentro de estas tendencias hay ejemplos clamorosos de excepcionalidad: en la segunda década El amor en vizcaíno (1615) tiene un 61,9\% de romance; en la tercera década El primer conde de Orgaz (1623) tiene un $10,7 \%$.

Por tanto, salvo que El alba y el sol forme parte de esas excepciones, el uso del romance nos marca como fecha de escritura esa tercera década de siglo.

El alba y el sol tiene 242 versos en redondillas (7,6\%). Es la pieza dentro del corpus que trabajamos que presenta el porcentaje más bajo. En este sentido es claramente una pieza extraña pues aunque el uso de este metro es muy irregular en Luis Vélez, oscila entre el 30 y el $50 \%$.

En la distribución del uso de romances y redondillas en el corpus velecista observamos que a partir de 1615 se empieza a imponer el primero sobre el segundo.?

En cuanto a los versos sueltos, en las piezas de Luis Vélez van desapareciendo, al tiempo que aumentan los pareados en sueltos. $\mathrm{Y}$ comienza a tener una presencia significativa la silva.

\begin{tabular}{|l|l|c|c|c|}
\hline \multicolumn{1}{|c|}{ FECHA } & \multicolumn{1}{|c|}{ TÍTULO } & \% sueltos & $\%$ par. en su. & $\%$ silva \\
\hline $1597 ?-1602$ & Prodigioso príncipe transilvano & 6,5 & 0 & 0 \\
\hline $1608-1610$ & Los fijos de la barbuda & 8,8 & 0,8 & 0 \\
\hline 1613 & La serrana de la Vera & 8,3 & 23,6 & 0 \\
\hline
\end{tabular}

6 Cfr. R. Bininger y R. L. Landeira, op. cit., p. 29.

7 Lógicamente también va acompañado de excepciones como la de El conde don Pero Vélez (1615): rom 29\% y red 65\%; El primer conde de Orgaz (1623): rom 10,7\% y red 47,5\% y El cerco del Peñón de Vélez (1634): rom $24,9 \%$ y red $67,5 \%$. 


\begin{tabular}{|l|l|c|c|c|}
\hline \multicolumn{1}{|c|}{ FECHA } & \multicolumn{1}{|c|}{ TÍTULO } & \% sueltos & $\%$ par. en su. & $\%$ silva \\
\hline $1615-17$ & El conde don Sancho Niño & 2,8 & 33,8 & 0 \\
\hline $1622-25$ & El Hércules de Ocaña & 2,1 & 42,8 & 3,4 \\
\hline $1630 ?-39 ?$ & Reinar después de morir & 0 & - & 13,8 \\
\hline
\end{tabular}

No hay versos sueltos en El alba y el sol. Tampoco se encuentran pareados sueltos. Sí contamos hasta ciento noventa y seis versos en silva $(6,2 \%)$. El porcentaje nos dirige de nuevo hacia la década de los veinte y más concretamente hacia su segunda mitad.

Muchas de las obras de Luis Vélez posteriores a 1620 tienen el final de sus actos en romance. ${ }^{8}$ Así ocurre con los finales de El alba y el sol: todos acaban en romance.

Otro posible factor para la fijación de la fecha es el parecido con obras como La vida es sueño: en las dos hay caída del caballo, en las dos se sitúa al comienzo de la representación, en las dos se utiliza la misma versificación en ese fragmento.

El alba y el sol comienza con la caída de Pelayo del caballo (vv. 1-16), al igual que le ocurrió a Rosaura en La vida es sueño:

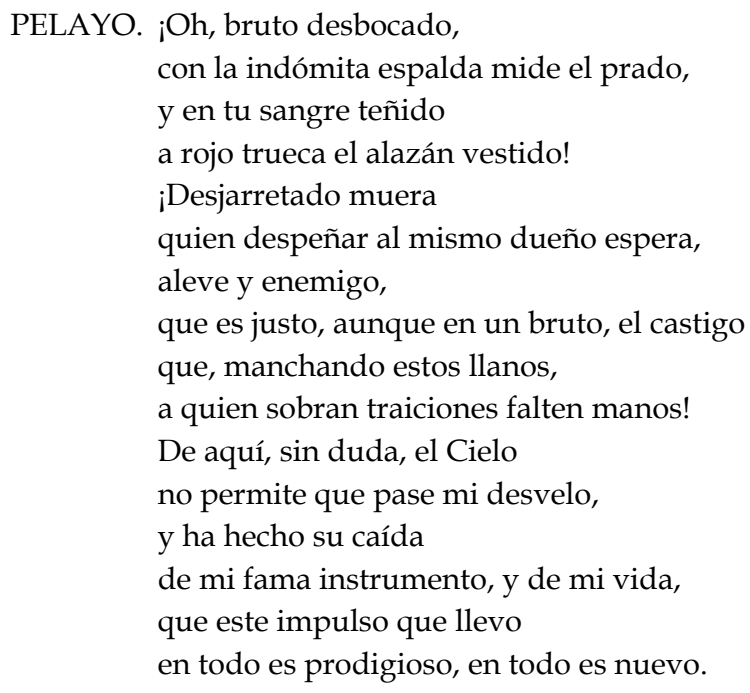

Como se puede observar coincide también con el fragmento de La vida es sueño en la versificación, silvas de consonantes o pareadas.

\footnotetext{
8 Cfr. Ibidem, p. 30. Recientemente R. Minian de Alfie, «Estudio introductorio», en El hijo del águila, de Luis Vélez de Guevara, Juan de la Cuesta, Delaware, 2003, p. 16, ha matizado tanto el estudio que estamos citando de R. Bininger y R. L. Landeira como la edición que hace R. Rozzell [1959] de La niña de Gómez Arias. Afirma que no es posible sostener la rotundidad del final de actos en romance de Luis Vélez pues existen casos -como el de El hijo del águila- en que no se cumple.
} 
Datos históricos y bibliométricos del corpus de Luis Vélez de Guevara para la fecha de escritura de El alba y el sol

Según los diversos indicios métricos y de intertextualidad parece que El alba y el sol corresponde al tipo de escritura que desarrolló Luis Vélez en la época que va de 1625 a 1630.

También es posible que esta obra fuese una extrañeza dentro del contexto dramatúrgico de Luis Vélez. Al menos dos datos nos hacen pensar así: el enorme uso de romance y el escasísimo de redondilla. ${ }^{9}$

Contrastaremos ahora el marco temporal aportado con el estudio de las fuentes y el contexto histórico de su posible representación. La historia de la Cava y el rey Rodrigo se transmitiría fundamentalmente de forma oral. No hemos encontrado testimonios escritos, fuera de los romances que nos han llegado, que verifiquen los conocidos sucesos de estos dos personajes. Incluyo uno de esos romances: ${ }^{10}$

\begin{tabular}{|c|}
\hline $\begin{array}{l}\text { uidado, } \\
\text { do; } \\
\text { dosado: } \\
\text { ionado; } \\
\text { mandado; } \\
\text { ça o grado. } \\
\text { a tomado; } \\
\text { illado: } \\
\text { el vado; } \\
\text { do. } \\
\text { ado, } \\
\text { entado; } \\
\text { biado. } \\
\text { ue por grado } \\
\text { do, } \\
\text { aviado, }\end{array}$ \\
\hline
\end{tabular}

El siguiente dato histórico citado en El alba y el sol que puede conducirnos a la fuente de inspiración del poeta es el mandato de supresión de armas en el reino. Así aparece en los versos 1064-1073:
porque dende que Rodrigo
las armas mandó quitar, que le quiso Dios ciegar, para endonalle el castigo,

\footnotetext{
9 Otra singularidad de la pieza es que representa el acontecimiento histórico más alejado dentro del corpus de dramas de historia nacional. Dentro de las históricas de tema extranjero sólo se remontan a tiempos anteriores al siglo VIII Juliano Apóstata (s. IV) y Atila azote de Dios (s. V). Una tercera de ámbito no nacional es de la misma época: El cerco de Roma por el rey Desiderio (s. VIII).

10 J. de Timoneda, Rosas de romances (Valencia, 1573), Valencia, Castalia, 1963.
} 
como en la troj finca o trigo,

cuantas puede conquerir

las fiz dentro zambullir

cuantas pude conseguir

por mis manos, con que puedo,

por la Vera Cruz de Oviedo,

más de mil homes guarnir.

Pero este asunto del desarme nos conduce, no al rey Rodrigo y los años de la invasión, sino a Witiza, que cambió los útiles de guerra por instrumentos de labranza.

Hay otro elemento que se puede fácilmente rastrear: se trata de la historia que se cuenta en los vv. 1162-1207 sobre una casa con un arca que contiene un misterioso lienzo.

En este tiempo, en Toledo, por antigüedad notoria, cerrado estaba un Palacio con candados y espantosas cerraduras diferentes, y escritas de letras godas que ningún rey hasta entonces se atrevió por su persona, ni por los suyos, a abrille, por ser fama que de sombras encantadas era albergue que guardaban en sus rotas ruínas tesoros grandes, y que el rey que sus medrosas puertas abriese, de España perdería la Corona con España juntamente. Rodrigo, haciendo de todas Estas, a su parecer, supersticiones heroicas bizarrías al silencio, rompió aquella prodigiosa fábrica que tantos días veneró el miedo, y con poca atención, echó sus puertas por el suelo, hallando sola, en todo el palacio, un arca de madera misteriosa, y dentro de ella no más de un lienzo, de estrañas formas de hombres pintados, y trajes de colores a la mora usanza, con unas letras que en antigua lengua goda decían: "Por esta gente tan soberbia y espantosa 


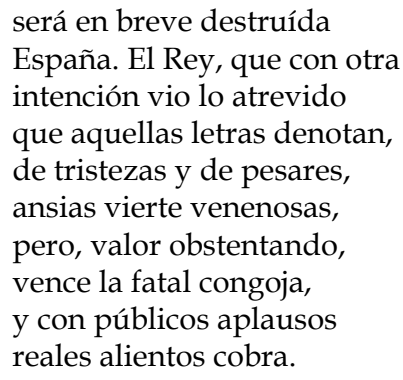

Debía tratarse de una de tantas leyendas que circulaban de boca en boca y nadie se detenía a escribir. Sólo hemos hallado una fuente escrita que la recoja, pero es muy posterior a la escritura de El alba y el sol. Feijoo menciona un palacio de Toledo en su Teatro crítico universal, concretamente en el discurso séptimo que versa sobre las cuevas de Salamanca y Toledo. Dice así:

aquel Palacio encantado, que dice el Arzobispo D. Rodrigo había en Toledo, y estaba siempre cerrado por no sé qué predicción creída, de que cuando se abriese, se perdería España; pero el infeliz Rey D. Rodrigo le mandó abrir, y entrando en él, halló un lienzo en que estaban pintados hombres armados de hábito, y gesto de Moros, con esta inscripción: Por esta gente será en breve destruida España. Digo que algunos creen, que aquel Palacio encantado no era otro, que la Cueva de que hablamos. ${ }^{11}$

Este palacio se identifica hoy en día en Toledo con el mismo lugar en que estaría emplazada la mítica cueva de Hércules. Al terminar la calle de Los Bécquer está, a la izquierda, el callejón de San Ginés. Al final de este callejón estaría el lugar donde el rey don Rodrigo vio las pinturas que relataban la invasión musulmana y la pérdida de España.

La búsqueda de fuentes para los personajes fue infructuosa en el caso de Alba. Sin embargo, del personaje Sando aparecen muchas referencias que parecen tomadas de la Crónica del ínclito emperador de España don Alonso VII...: sacada de un libro muy antiguo escrito de mano con letras de los godos, por relación de los mismos que lo vieron, y de muchas escrituras y privilegios originales del mesmo emperador y otros, de Fray Prudencio de Sandoval. Muy probablemente, esta crónica, junto a las leyendas que ya conocía Luis Vélez, fueron las fuentes de El alba y el sol. Esta crónica va dedicada al duque de Lerma, padre de aquel a quien sirviera el dramaturgo durante más de una década, por lo que le resultaría sencillo acceder a ella. Reproduzco el fragmento de la crónica por su interés y cercanía a lo que el drama refleja:

${ }_{11}$ Feijoo, Teatro crítico universal, Tomo séptimo, Discurso séptimo, «Cuevas de Salamanca, y Toledo, y Mágica de España», 1736. 
afirmase que hay papeles de antigüedades de España en poder de don Pedro Fernández de Velasco, condestable de Castilla, que fue príncipe muy curioso: y en ellos se decía, que en los tiempos muy antiguos estos caballeros se llamaban Sando Cuervo, y que eran del linaje de los godos: y en su tiempo, antes que España se perdiese, fueron señores de aquel famoso lugar de Amaya, Villasandino, con otros muchos de aquella comarca, y tan exentos, y libres en su señorío, que labraban moneda: y cuando se perdió España se juntaron a la defensa de ella con el infante don Pelayo, y fueron los principales en levantarlo por Rey, y hacer guerra a los moros. Y sucedió que como un día en una sangrienta refriega los nuestros, por ser infinitos los enemigos, se viesen vencidos, retiráronse a las montañas, que era su común y más segura guarida, y los moros los iban siguiendo fieramente, metiéronse por lo estrecho de unos montes, donde esta Covadonga, porfiando los enemigos en seguirlos: y sin duda les fuera muy mal, si un valiente caballero, llamado Sando Cuervo, no le hiciera rostro con sus grandes fuerzas, y valentía. Atravesó en el estrecho camino un gran madero, de los muchos que en aquellas montañas siempre hay caídos, y con sola su espada y escudo, solo se puso a pelear con los moros, defendiendo con el madero el paso, y los detuvo tanto, que aunque los enemigos rompieron el madero, y mataron a este valiente caballero, el rey don Pelayo tuvo lugar de recoger todos los suyos, y ponerlos en orden y volver a la pelea; en la cual con el favor del cielo vencieron, y mataron muchos moros. Acabada la contienda, viendo el rey don Pelayo el cuerpo de Sando Cuervo hecho pedazos, y como por su valor tenían las vidas, lloró mucho su muerte con palabras de mucho sentimiento, diciendo entre ellas: O Sando Cuervo, que no has sido sino Sando Vale, pues con solo tu brazo poderoso has sido hoy nuestro Salvador; y tal debe ser tu nombre y de tus descendientes. Traían por divisas estos caballeros antes dello un Cuervo coronado en campo de oro, y que en lugar del Cuervo pusieron la viga atravesada, que es la banda negra ahora traen. ${ }^{12}$

En el Libro de grandezas y cosas memorables de España hace referencia el cronista Pedro Medina a lo dramatizado en El alba y el sol. En el capítulo XXI trata «de cómo don Pelayo fue alzado por rey y, ayudado con el favor divino, comenzó a recobrar España: de las victorias grandes que de los moros hubo y de la muerte del conde Julián y de los otros traidores». ${ }^{13}$

Otra característica de El alba y el sol permite conjeturar la fecha de escritura. Se trata del carácter genealógico. En diversos diálogos se deja entrever el deseo de adular a determinada familia bien citando una hazaña de un antepasado suyo bien haciendo referencia al origen de sus apellidos o armas heráldicas.

La adulación a esa familia a través de los diálogos y acciones constituye el punto de cruce de la composición de la obra teatral y las circunstancias históricas del momento en que se escribió.

12 P. Sandoval, Crónica del ínclito emperador de España don Alonso VII, Madrid, publicada por Luis Sánches, 1960, fols. 189-190.

13 P. Medina, Obras de Pedro Medina (Libro de grandezas y cosas memorables de España y Libro de la verdad), ed. y prólogo de Ángel González Palencia, Madrid, CSIC, 1944, pp. 35-36. Para el resto de referencias históricas y literarias sobre los hechos dramatizados dirijo al «Estudio introductorio» de Profeti [2010], pp. 18-23. 
Uno de los primeros pasajes en los que se intuye el tono de alabanza genealógica es el que hace referencia a la hazaña de Sando en su batida contra los moros. Los versos 2258-2274 lo recogen así:

PELAYO. ¿Qué es esto, Sando?

\begin{tabular}{|c|c|}
\hline ANDO. & $\begin{array}{l}\text { Pelayo, } \\
\text { a esto de español me obliga } \\
\text { el valor. }\end{array}$ \\
\hline PEL & ¿Cómo? \\
\hline SANDO. & $\begin{array}{l}\text { Esta viga, } \\
\text { que ya en mis brazos es rayo, } \\
\text { de un edificio arranqué, } \\
\text { que, deshecho y derribado, } \\
\text { sobre esas peñas, cansado } \\
\text { de haberse tenido en pie, } \\
\text { contra el tiempo se arrojó. }\end{array}$ \\
\hline
\end{tabular}

PELAYO. ¿Qué es lo que con ella intentas?

ALBA. A la edad pasada, afrentas que esta lealtad no alcanzó.

PELAYO. ¿De qué suerte?

SANDO. Si has oído los moriscos instrumentos, de mis altos pensamientos el blasón nunca vencido verás agora.

Vase con la viga.

Este diálogo recuerda una memorable acción pasada y narra lo que probablemente en la representación no se captaría con todo detalle. El dramaturgo ha querido subrayar esta acción con la palabra y, muy probablemente, la representación de Sando haciendo retroceder a los moros con el solo uso de una viga. Aunque también pudo recurrir Luis Vélez a la ticoscopia, técnica que utiliza en otras obras. ${ }^{14}$

La primera pista seguida para descubrir las intenciones del fragmento anterior fue la viga. Por la importancia que en él se le da parecía lógico poder encontrarlo en el escudo de alguna familia noble. En heráldica el palo o viga es el jeroglífico de la lanza del Caballero o de las estacas que llevaban los soldados a la guerra para cerrar los campamentos o de una especie de viga de las armerías que los nobles hacían montar delante de su tienda o del puente levadizo de su morada, como señal de jurisdicción. Este elemento común en los escudos de muchas familias abría en exceso el campo de investigación y dificultaba sobremanera que se encontrase una única familia con este distintivo.

14 Vid. J. J. González Martínez, «Ticoscopia, espacio y tiempo de los torneos caballerescos en Luis Vélez», en Felipe B. Pedraza, Rafael González Cañal y Gemma Gómez Rubio (eds.), Espacio, tiempo y género en la comedia española. Actas de las II Jornadas de Teatro Clásico, Toledo, 14-16 de noviembre de 2003, Almagro, UCLM, 2005, pp. 77-78. 
Posteriormente conocimos la historia del conde Fernando Negro, cuya similitud con la vida de Sando Cuervo hace creer que se trata de la misma persona. Fernando Negro se unió a don Pelayo desde los mismos inicios de la Reconquista, fue uno de los nobles juramentados en Covadonga y en la primera batalla ganada a los moros fue uno de los héroes más destacados.

Conocemos muy poco de la batalla de Covadonga teniendo en cuenta que fue la primera ocasión en la que se ponía freno al avance musulmán. Ninguna de las crónicas musulmanas de la época que han llegado a nuestras manos recoge nada sobre esta batalla de Covadonga.

Un paréntesis historiográfico semejante nos encontramos en las crónicas cristianas de esa época, en las que tampoco se habla apenas de Pelayo. Será necesario esperar a Alfonso III de León (866-910) para tener noticias de lo que pudo ocurrir en aquel momento. En tiempos de este rey se redactan unas crónicas llamadas «Albedense» y la de Alfonso III. ${ }^{15}$ El primer objetivo de dichas crónicas era establecer una continuidad entre la monarquía visigoda y el reino de Asturias.

Las diferencias de estas crónicas con la forma de reflejar la historia en la actualidad hicieron que se minusvaloraran por el aparente exceso de fábula, novela y leyenda. Esto provocó que desde el siglo XVIII se pusiera en duda la autenticidad de esta batalla e incluso del mismo Pelayo. Finalmente, la crítica histórica del profesor Sánchez Albornoz fijó de manera sobria los sucesos de Covadonga al definirlos como una escaramuza entre dos grupos relativamente poco numerosos.

En esa batalla o escaramuza participó, por tanto, el conde don Fernando Negro, según unos: señor de la casa solar González en el valle de Jivaja, en la montaña de Santander, según otros: origen del linaje de los Sandoval. Y este es el indicio que más luz da a nuestro estudio, dada la relación de Luis Vélez con los Sandoval.

Diego Gómez de Sandoval y Rojas es el segundo hijo del primer duque de Lerma y hermano del duque de Uceda, ambos sucesivos favoritos de Felipe III. Este segundón casó en 1604 con Luisa de Mendoza, hija primogénita del primer matrimonio de Ana de Mendoza (y por ello condesa de Saldaña). Este matrimonio vino a favorecer a ambas familias, ya que para los Sandoval suponía emparentar con los poderosos Mendozas, mientras que para los del Infantado significaba lograr el apoyo del favorito en el pleito con sus primos. Aunque Luisa de Mendoza murió tempranamente, en 1619, dejando

15 Vid. J. Gil Fernández, Introducción y edición de Crónicas asturianas: Crónica de Alfonso III (Rotense y «A Sebastián»); Crónica Albeldense (y «Profética»), Oviedo, Universidad de Oviedo, 1985. 
Datos históricos y bibliométricos del corpus de Luis Vélez de Guevara para la fecha de escritura de El alba y el sol como fruto de esa unión un heredero, su marido conservó hasta su muerte el título de conde de Saldaña. ${ }^{16}$

De la época en que Luis Vélez sirvió al conde tenemos la mayor parte de los escasos datos biográficos del dramaturgo. Comienza esa etapa junto a Diego Gómez de Sandoval y Rojas en el año $1603 .{ }^{17}$ Le sirve como gentil hombre de cámara, como sabemos por el opúsculo que en 1608 tituló Elogio del Juramento del Serenísimo Príncipe don Felipe Domingo, Cuarto de este nombre, en el que por primera vez, firma como Luis Vélez de Guevara y se declara criado del conde de Saldaña.

También sabemos que en 1608 «Lope es requerido por el conde de Saldaña para solucionar un enfado entre él y Luis Vélez. Lope [...] defiende a Vélez y envía junto con la carta un poemilla al conde en que encubiertamente menciona las relaciones entre Belardo (Lope), Salicio (Saldaña) y Vélez (Lauro), Epistolario, t.III, pp. 8-9».18

En septiembre de 1608, el conde de Saldaña le otorga la cantidad de cuatrocientos ducados «de ayuda de costa por una vez y a doña Úrsula Bravo, con quien está concertado de casarse, criada que ha sido de mi señora la marquesa de Alcañices». ${ }^{19}$

La siguiente noticia de carácter laboral es del veinte de noviembre de 1619: en la partida de defunción de su mujer aparece Luis Vélez como criado del Marqués de Peñafiel, en cuyo servicio estuvo cerca de dos años. ${ }^{20}$

\footnotetext{
16 Diego Gómez de Sandoval y Rojas, conde de Saldaña, es hijo del duque de Lerma y de doña Catalina de la Cerda. Dice J. Entrambasaguas, «Un olvidado poema de Vélez de Guevara», Revista de Bibliografía Nacional, tomo 2 (1941), p. 98: «según diversos testimonios históricos, muy inteligente, bello de rostro y de agradable apostura, unido todo a un carácter simpático y atrayente; pero de vida tan disipada, por su desenvoltura moral, que resultaba un loco de atar, lo cual no impedía que su padre tuviera por él particular afección. Afligido el poderoso valido de Felipe III por la manera de ser de D. Diego, creyó corregirle con el matrimonio y logró casarle, el 30 de agosto de 1603, en Valladolid, con D ${ }^{a}$ Luisa de Mendoza, IV duquesa del Infantado, siendo padrinos de bodas los Reyes y dándose una gran fiesta con tal motivo. Pero el nuevo conde de Saldaña, que hubo de trocar su apellido familiar por el de Hurtado de Mendoza -según exigencia de los esponsales para que percibiera el matrimonio las rentas del mayorazgo de la Condesa- no varió, en cambio su modo de ser y de vivir, dando en mil desordenadas locuras que fueron causa de que le hirieran en una ocasión y, al fin, motivo más que suficiente para que su padre, dejando a un lado su cariño y preferencias por D. Diego, le recluyera durante cierto tiempo en la fortaleza de Ampudia, castillo próximo a Valladolid, lo cual frenó bastante su desorbitada conducta».

17 Cfr. T. Ferrer [1991], op. cit., p. 138.

18 Ídem. Además es interesante consultar R. González Cañal, «Lope, la corte y los pájaros nuevos», Anuario Lope de Vega, 8 (2002), pp. 139-162, donde se analizan las relaciones del Fénix con el poder, su falta de reconocimiento en la Corte y su trato con los jóvenes dramaturgos que sí triunfaron en Palacio.
}

19 Documento transcrito por C. Pérez Pastor, Bibliografía madrileña o descripción de las obras impresas en Madrid, Madrid, Tipografía de la Revista de Archivos, Bibliotecas y Museos, 1906-1907, III, p. 500, citado en El diablo Cojuelo: glosario e índices léxicos, tesis doctoral inédita de D. Azorín Fernández.

20 Para la relación de Luis Vélez de Guevara con la corte, los Sandoval, los Téllez-Girón y Olivares puede consultarse: G. A. Davies, «Luis Vélez de Guevara and Court Life», en C. George Peale (ed.), Antigüedad y actualidad de Luis Vélez de Guevara: estudios críticos, Amsterdam, Benjamins, 1983. 
Pero la referencia a los Sandoval no se queda sólo en la anécdota de la viga. En los vv. 2670-2672 cuenta el origen del apellido de la noble familia:

PELAYO. ¡Sando, válgame tu brazo!

SANDO. ¡Solo ese apellido quiero

para mi sangre, Pelayo!

Hemos visto antes cómo unas fuentes marcan como origen de los Sandoval a un tal Fernando Negro. Otras fuentes denominan a este mismo personaje como Sando Cuervo que murió al cerrar el paso con una viga a los moros que acechaban al rey Pelayo. Debió ser entonces cuando el rey pronunciase las palabras que después quedarían de apellido a la familia que cambió el Sando Cuervo por Sando Vale. También en memoria de este hecho, sus sucesores cambiaron en su escudo la figura del cuervo sobre campo de oro por una viga azul. Todo ello está fundamentado en las páginas que el cronista fray Prudencio dedica a la casa de Sandoval en la obra citada más arriba.

Por tanto, según los datos históricos, El alba y el sol debió de escribirse después de 1605, año en que comienza a servir al conde de Saldaña ${ }^{21}$. También sabemos que debió de ser escrita antes de 1618, que es cuando abandona al conde de Saldaña. ${ }^{22}$ Pero no llegó a separarse del todo de los Sandoval en 1618 ya que su nuevo señor, el marqués de Peñafiel, ${ }^{23}$ estaba casado con Isabel de Sandoval y Rojas, séptima hija del duque de Lerma y hermana por tanto del conde de Saldaña don Diego. A este nuevo señor sirvió cerca de dos años. Por los datos históricos hasta aquí obtenidos podríamos pensar que la obra se escribiría entre los años 1605 y $1620 .{ }^{24} \mathrm{El}$ tono del drama es más bien de carácter aúlico por lo que podríamos fijar el lugar de representación en un espacio cortesano.

Hasta aquí hemos ofrecido los resultados de la investigación a través de la aplicación del método de Morley y Bruerton y a través del contexto histórico de escritura. Ambos márgenes de composición difieren. Nos inclinamos por los criterios de oportunidad histórica ya que sería extraño el apoyo a los Sandoval después de 1621, tras la llegada al poder de Felipe IV y el conde-duque. Además -como ya hemos destacado- la pieza contiene una serie de irregularidades métricas que dificultan su datación por el estilo de escritura.

\footnotetext{
${ }_{21}$ E. Cotarelo [1916], op. cit., p. 639.

22 Cfr. G. A. Davies, op. cit., p. 24.

${ }^{23}$ Juan IV Téllez-Girón y Enríquez de Ribera, 1597-1656, cuarto duque de Osuna, G.E., tercer marqués de Peñafiel, octavo conde de Ureña. Casa con Isabel Sandoval y Rojas el once de diciembre de 1617.

${ }^{24}$ Me parece que debemos considerar como límite posible de escritura el año 1620, y no 1618, pues es el final de la relación directa del dramaturgo con los Sandoval. Además la caída de Lerma en 1618 no es el final de los Sandoval pues como es conocido le continúa en el valimiento su hijo el duque de Uceda, Cristóbal Gómez de Sandoval y Rojas.
} 


\section{Bibliografía}

AZORÍN FERNÁNDEZ, D., El diablo Cojuelo: glosario e índices léxicos, tesis doctoral inédita, sl.

BOLAÑOS, P., «Introducción», La serrana de la Vera de Luis Vélez de Guevara, Madrid, Castalia, 2001, pp. 9-94.

BIANCO, F. J., «Introducción», en Más pesa el rey que la sangre de Luis Vélez de Guevara, Barcelona, Puvill-Editor, 1979.

BININGER, R., y R. L. LANDEIRA, «Introducción», en El Conde don Sancho Niño de Luis Vélez de Guevara, Vigo, Faro de Vigo, 1970, pp. 9-50.

BRUERTON, C., «The date of Schaeffer's Tomo Antiguo», Hispanic Review, XV (1947), p. 346-364.

BRUERTON, C., «Eight plays by Vélez de Guevara», Romance Philology, vol. VI, nº 4 (mayo 1953), pp. 248-253.

COTARELO, E., «Luis Vélez de Guevara y sus obras dramáticas», BRAE, III (1916), pp. 621-652.

COTARELO, E., «Luis Vélez de Guevara y sus obras dramáticas», BRAE, IV (1917), pp. 269-308.

DAVIES, G. A., «Luis Vélez de Guevara and Court Life», en C. George Peale (ed.), Antigüedad y actualidad de Luis Vélez de Guevara: estudios críticos, Amsterdam, Benjamins, 1983, pp. 20-38.

ENTRAMBASAGUAS, J., «Un olvidado poema de Vélez de Guevara», Revista de Bibliografía Nacional, tomo 2 (1941), pp. 91-176.

FERRER VALLS, T., La práctica escénica cortesana: de la época del emperador a la de Felipe III, London, Tamesis Book, 1991.

FERRER VALLS, T., Nobleza y espectáculo teatral (1535-1622), Valencia, UNEDUniversidad de Sevilla-Universitat de València, 1993.

GIL FERNÁNDEZ, J., Introducción y edición de Crónicas asturianas: Crónica de Alfonso III (Rotense y «A Sebastián»); Crónica Albeldense (y «Profética»), traducción y notas de José L. Moralejo; estudio preliminar de Juan I. Ruiz de la Peña, Oviedo, Universidad de Oviedo, 1985.

GONZÁLEZ CAÑAL, R., “Lope, la corte y los pájaros nuevos”, Anuario Lope de Vega, 8 (2002), pp. 139-162.

GONZÁLEZ MARTÍNEZ, J. J., Tesis doctoral inédita El teatro histórico nacional de Luis Vélez, Universidad de Valladolid, 2007.

GONZÁLEZMARTÍNEZ,J.J., «Ticoscopia, espacio y tiempo de los torneos caballerescos en Luis Vélez», en Felipe B. Pedraza, Rafael González Cañal y Gemma Gómez Rubio (eds.), Espacio, tiempo y género en la comedia española. Actas de las II Jornadas de Teatro Clásico, Toledo, 14-16 de noviembre de 2003, Almagro, UCLM, 2005, pp. 
73-92.

GONZÁLEZ MARTÍNEZ, J. J., «Estudio introductorio», en Si el caballo vos han muerto, y blasón de los Mendozas de Luis Vélez de Guevara, George Peale y William R. Manson (eds.), Delaware, Juan de la Cuesta, 2007, pp. 13-21.

GONZÁLEZ MARTÍNEZ, J. J., «El rey como espectador del teatro indiano: Las palabras a los reyes de Luis Vélez de Guevara», en Sonia Mattalia, Pilar Celma, Pilar Alonso (eds.); Anna Chover Lafarga, Carmen Morán Rodríguez (colaboradoras), Actas del VII Congreso Internacional de la AEELH-Universidad de Valladolid, Homenaje a José Luis de la Fuente, «El viaje en la Literatura Latinoamericana: El espíritu colombino», Valladolid 19-22 de septiembre de 2006; Frankfurt am Main, Vervuert, Iberoamericana, 2008, pp. 213-233.

GONZÁLEZ MARTÍNEZ, J. J., «Más pesa el rey que la sangre: el Guzmán de Luis Vélez junto al Quijote y el Cid», en J. Matas Caballero y J. M. Balcells Doménech (eds), D. Pérez Fernández (coord.), Cervantes y su tiempo, Actas del Congreso celebrado en León 2-5 de noviembre de 2005, vol. I, Lectura y Signo, Anejo I, León, Universidad de León, 2008, pp. 439-448.

GONZÁLEZ MARTÍNEZ, J. J., «La mujer fuerte y vengadora en el drama históriconovelescos de Luis Vélez de Guevara: La montañesa de Asturias», en Actas del II Congreso Internacional Imagen y Palabra de Mujer, Valladolid, 10-12 de mayo de 2006, Junta de Castilla y León, Instituto de la Lengua, 2009, pp. 175-184.

GONZÁLEZ MARTÍNEZ, J. J., «Los motivos de la censura civil de La mayor desgracia de Carlos Quinto, de Luis Vélez de Guevara», en Actas del XIV Congreso de la Asociación Internacional de Teatro Español y Novohispano de los Siglos de Oro, Olmedo, 20-23 de julio de 2009, Valladolid, Universidad de ValladolidAyuntamiento de Olmedo, 2010, pp. 563-572.

GONZÁLEZ MARTÍNEZ, J. J., «Estudio introductorio», en El asombro de Turquía de Luis Vélez de Guevara, George Peale y William R. Manson (eds.), Delaware, Juan de la Cuesta, 2010, pp. 13-26.

HAUER, M. G., Luis Vélez de Guevara: A critical bibliography, North Carolina, University of North Carolina Press, 1975.

HAUER, M. G., «An addendum to Luis Vélez de Guevara: A critical bibliography», en George Peale (ed.), Antigüedad y actualidad de Luis Vélez de Guevara: Estudios críticos, Amsterdam, Benjamins, 1983, pp. 254-298.

HERZOG, W., Introducción y edición de la Comedia famosa del rey don Sebastián, Madrid, Anejos del Boletín de la Real Academia Española, 1972.

MEDINA, P., Obras de Pedro Medina (Libro de grandezas y cosas memorables de España y Libro de la verdad), ed. y prólogo de Ángel González Palencia, Madrid, CSIC, 1944.

MENÉNDEZ PIDAL, R., Y M. GOYRI DE MENÉNDEZ PIDAL, «Introducción», en La 
Datos históricos y bibliométricos del corpus de Luis Vélez de Guevara para la fecha de escritura de El alba y el sol

serrana de la Vera, Teatro Antiguo Español, I, Madrid, 1916.

MINIAN DE ALFIE, R., «Estudio introductorio», en El hijo del águila, de Luis Vélez de Guevara, George Peale y William R. Manson (eds.), Delaware, Juan de la Cuesta, 2003, pp. 13-37.

MORLEY, S. G., Y C. BRUERTON, Cronología de las comedias de Lope de Vega, versión española de M. R. Cartes, Madrid, Gredos, 1968.

PEALE, G., «Estudio bibliográfico y métrico», en El espejo del mundo de Luis Vélez de Guevara, Delaware, Juan de la Cuesta, 2002, pp. 85-120.

PEALE, G., «Estudio introductorio», en La mayor desgracia de Carlos Quinto de Luis Vélez de Guevara, Delaware, Juan de la Cuesta, 2002, pp. 39-125.

PEALE, G., «Estudio introductorio», en El conde don Pero Vélez de Luis Vélez de Guevara, Juan de la Cuesta, Delaware, 2002, pp. 37-86.

PEALE, G., «Estudio introductorio», en El amor en vizcaíno, celos en francés y torneos de Navarra de Luis Vélez de Guevara, Delaware, Juan de la Cuesta, 2002, pp. 109125.

PEALE, G., «Estudio introductorio», en El primer conde de Orgaz de Luis Vélez de Guevara, Delaware, Juan de la Cuesta, 2002, pp. 14-88.

PEALE, G., «Estudio introductorio», en El águila del agua de Luis Vélez de Guevara, Delaware, Juan de la Cuesta, 2003, pp. 13-82.

PEALE, G., y H. URZÁIZ TORTAJADA, «Vélez de Guevara», en Historia del Teatro Español, Madrid, Gredos, 2003, pp. 929-959.

PEALE, G., «Estudio introductorio» en El hijo del águila de Luis Vélez de Guevara, Delaware, Juan de la Cuesta, 2003, pp. 41-51.

PEALE, G., «Estudio textual y criterios de edición», en El cerco del Peñón de Vélez de Luis Vélez de Guevara, Delaware, Juan de la Cuesta, 2003, pp. 47-53.

PEALE, G., «Estudio introductorio», en El rey naciendo mujer de Luis Vélez de Guevara, Delaware, Juan de la Cuesta, 2006, pp. 31-42.

PEALE, G., «Estudio bibliográfico», en Si el caballo vos han muerto, de Luis Vélez de Guevara, Delaware, Juan de la Cuesta, 2007, pp. 31-39.

PEALE, G., «Estudio bibliográfico y métrico», en Reinar después de morir de Luis Vélez de Guevara, Delaware, Juan de la Cuesta, 2008, pp. 41-54.

PEALE, G., «Vélez de Guevara contextualizado: una vida singular y su ámbito ético», Bulletin of the Comediantes, vol. 61, nº 1 (2009), pp. 51-96.

PEALE, G., «Estudio bibliográfico», en El asombro de Turquía de Luis Vélez de Guevara, Delaware, Juan de la Cuesta, 2010, pp. 27-49.

PEALE, G., «Estudio bibliográfico», en El alba y el sol, de Luis Vélez de Guevara, Delaware, Juan de la Cuesta, 2010, pp. 39-82.

PROFETI, M. G., «Introduzione, testo critico e note», en Virtudes vencen señales de Luis Vélez de Guevara, Pisa, Universitá di Pisa, 1965, pp. 5-61. 
RODRÍGUEZ CUADROS, E., «Introducción», en El amor en vizcaíno, celos en francés y torneos de Navarra de Luis Vélez de Guevara, Delaware, Juan de la Cuesta, 2002, pp. 13-107.

ROZZELL, R., «Introducción», en La niña de Gómez Arias de Luis Vélez de Guevara, Granada, Universidad de Granada, 1959, pp. 7-30.

SANDOVAL, P., Crónica del ínclito emperador de España don Alonso VII, publicada por Luis Sánches, Madrid, 1960.

SPENCER, F. E., Y R. SCHEVILL, The dramatics works of Luis Vélez de Guevara. Their plots, sources, and bibliography, Berkeley, University of California Press, 1937.

TIMONEDA, J. de, Rosas de romances (Valencia, 1573), Castalia, Valencia, 1963.

URZÁIZ TORTAJADA, H., Luis Vélez de Guevara, Teatro Breve, Madrid, IberoamericanaVervuert, 2002.

VÉLEZ DE GUEVARA, L., El alba y el sol; con partituras de Antonio Guerrero (1761) y Enrique Moreno (1827); ed. crítica y anotada de William R. Manson y C. George Peale, estudio introductorio de Maria Grazia Profeti, Delaware, Juan de la Cuesta, 2010.

ZIOMEK, H., Y A. N. HUGHES, «A critical edition», en El cerco de Roma por el rey Desiderio de Luis Vélez de Guevara, Kassel, Edition Reichenberger, 1992, pp. 1-43.

ZUGASTI, M., «Estudio introductorio», en Las palabras a los reyes y gloria de los Pizarros, W. R. Manson y George Peale (eds.), Delaware, Juan de la Cuesta, 2004, pp. 49-86. 